\title{
Modelling Planar Electromagnetic Levitation System based on Phase Lead Compensation Control
}

\author{
Mundher H. A. YASEEN \\ Electrical \& Electronics Engineering Department \\ University of Gaziantep \\ Gaziantep, Turkey
}

\begin{abstract}
Electromagnetic Levitation System is commonly used in the field of train Maglev (magnetic levitation) system. Modelling Maglev system including all the magnetic force characteristics based on the current and position. This paper presents 2DOF model which represents a sample of uniform rigid plane body based on the functions of current and the air gap. The present work identifies the dynamic correlation of the levitation system of the Maglev using three sub-models. Lead controller is developed to achieve system stability by considering the system correlation of system moments and inductance variations. The control properties of the present model are obtained through SIMLAB microcontroller board to achieve the stable Maglev system.
\end{abstract}

Keywords-Electromagnetic levitation system; lead controller; (magnetic levitation) maglev system; SIMLAB board

\section{INTRODUCTION}

Magnetic levitation (Maglev) is one of the Intelligent Transportation Systems (ITS) scopes. It has become the greatest technology in the railways infrastructure domain. There are three types of Maglev technology: Electromagnetic suspension system (EMS), Electrodynamics suspension system (EDS) and Inductrack technology. Maglev fastest growing is consider as a strategic priority in advancing automation that is leading the global advocate for the benefits of automating [1]. Maglev systems can be monorail or dual rail [2] with three types of Maglev technology: Electromagnetic suspension system (EMS), Electrodynamics suspension system (EDS) and Inductrack. This research is used to analyse the effective issues of magnetic levitation due to the gap distance and evaluate its technical aspects based on the EMS of two degrees-of-freedom suspension system model. The challenge in this research is whether the available models could be realistically functional with unique properties. Many researchers developed the Electromagnetic suspension system (EMS), Al-Muthairi and Mzribi (2004) developed variation of system parameter scheme. They highlighted three different types of Maglev sliding mode controller: static SMC, dynamic SMC and modified dynamic SMC. The results observed that the modified dynamic SMC gave the best results among them [3]. Banerjee et al. (2007), designed and controlled single-axis EMS system. The nonlinear system was controlled based on nonlinear feedback linearizing technique. In the present system, the feedback linearization control supply a wide variation of operation point comparing with the linear one. The results observed a rejected controller of both disturbances successfully [4]. Lee et al. (2008), developed a novel control model to suspend objects based on exerted magnetic control force. The magnetic force in this method was proportional to the suspended object position and the employed voltage to the electromagnet in the proposed model. A sliding mode controller was able to work based on the proposed model for a lab built magnetic system. The results were more effective and robust against mass variation than the traditional controller adopting the conventional model [5]. Yousfi Khemissi (2010) developed SMC approach depends on sliding mode for controlling magnetic suspension system. The performance of this method was good for different disturbance signals in terms of trajectory tracking signal builder, pulse generator and random reference applied to the system. The load variations and the designed controller system stability provide a dynamic response which is considered a valid alternative for standard control methods like speed control and position. SMC considered as a good controller performance in disturbance attenuation [6]. Chunfang and Jian (2012) presented a novel second-order sliding mode controller to serve the electromagnetic levitation grip control. The presented design used in CNC (Computerized Numerical Control). The control strategy of the researchers was by using super-twisting algorithm in the design. The results observed a clear decrease in chattering effect of the slide model surface compared with traditional slide model and PID system control; therefore present system achieved a faster response and strong robustness [7]. Xing et al. (2014), presented multi-degrees of freedom system. The presented multivariable control system is nonlinear and strong coupling. The key part in this system was the linearization and decoupling of the levitation force which is used to achieve control with high speed and high precision. The results show the high speed response for the control system compared with a given reference; also the simulation observes the robustness due to disturbance [8]. Unni et al. (2016) design a PID, FUZZY and LQR control system. The systems implemented in real time using MATLAB software. The researchers compare the control system operations based on peak overshoot, rise time and settling time. The researchers recorded the features of each operation case [9]. Zhu et al. (2017) developed a six degree of freedom (6 DOF) magnetic levitation system. In this research the stator and translator are implemented by four groups. These groups involve 1-D Halbach permanent magnet (PM) arrays correlated with a set of coils. These components are controlling all the square coils (i.e. the eight-phase array). The results of these researches observed the root-mean-square [10]. It is seen from the present studies that the researchers always try to develop the control 
systems based on plate Maglev system using specific mathematical model to observe a unique controller as described.

\section{MAthematical MAgLEV System Model}

Mathematical Maglev System is modeled in order to analyze and predict the system response. The general description of the system considers a square flat plane with a dynamic distance dependent. The present system model proposes a novel Maglev system model which uses permanent magnets correlated with mechanism of plane control motion. The adjustment of permanent magnets' forces depends on the dynamic response of the angle of rotation of the plane and the air gap between the magnetic coil and the plane. For that the present model studies two degrees-of-freedom suspension system based on multiple forces. It begins with the formulation of three sub-models. These sub-models contain the mechanical sub-model, magnetic sub-model and electrical sub-model.

\section{A. Mechanical Sub-model}

The mechanical sub-model is the first step to investigate the Maglev system. The physical system consists of a flat plane tied with four discs that is to be levitated under four parallel electromagnet coils as shown in Fig. 1. The present 2DOF model represents a sample of uniform rigid car body, and it is suspended by four Maglev systems.

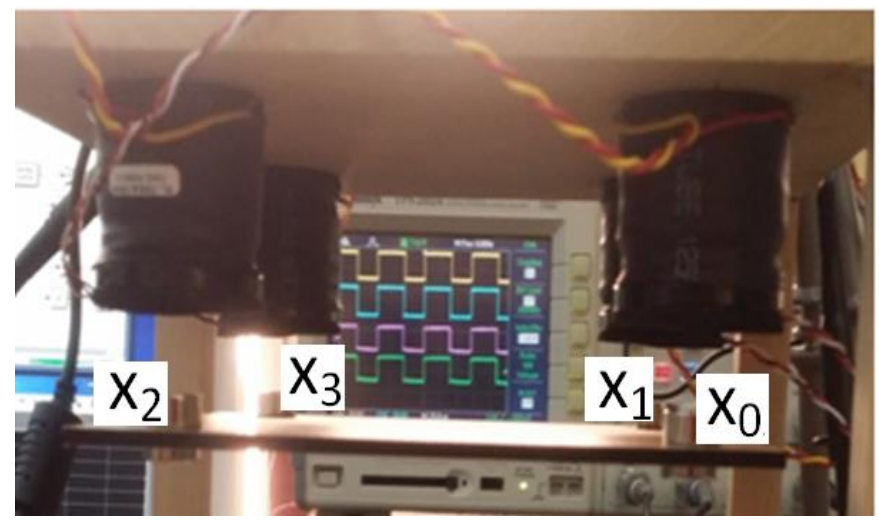

Fig. 1. Maglev physical system.

The levitation system involves four coils and magnet discs fixed on a flat plate in horizontal space which observes each disc position indicated by $\mathrm{X}_{0}, \mathrm{X}_{1}, \mathrm{X}_{2}$ and $\mathrm{X}_{3}$. The gravity force of the propose system modeled in the below equation:

$$
\begin{aligned}
& F_{\text {net }}=\sum_{1}^{i} F_{i}(t), \ldots(i=4) \\
& F_{\text {net }}(t)=F_{1}(t)+F_{2}(t)+F_{3}(t)+F_{4}(t)
\end{aligned}
$$

Where, $F_{1}, F_{2}, F_{3}$ and $F_{4}$ are the four forces which are produced by the electromagnets. The plane object moment around the $y$-axis define the effect of forces presented in (2) with respect to the distance from the y-axis to the disc position (d). The correlation between the disc position in term of air gap variation and the distance (d) can be expressed in (3).

$$
d=x \cdot \cos \theta
$$

Where, $\mathrm{x}$ is the air gap position and $\theta$ is the rotational motion of the plane. The moment of the body present in (4) and (5).

$$
\begin{aligned}
& M_{\text {net }}(t)=\sum_{1}^{i} M_{i}(t), \ldots(i=4) \\
& M_{\text {net }}(t)=M_{1}(t)+M_{2}(t)+M_{3}(t)+M_{4}(t) \\
& M_{\text {net }}(t)=d\left(F_{1}(t)+F_{2}(t)+F_{3}(t)+F_{4}(t)\right)
\end{aligned}
$$

The position of each disc in the system can be expressed in (7) below:

$$
x_{c}=x_{1}+x_{2}+x_{3}+x_{4}
$$

Considering the model as rigid body, (7) is calculating electromagnetic forces $F 1(t) F 2(t) F 3(t) F 4(t)$ (or voltages V1 (t), V2 (t), V3 (t) and V4 (t)) in accordance with three control variables $($ Fnet $(\mathrm{t})$, Mnet $(\mathrm{t}))$ required by the system. The indeterminacy present in this problem is removing by an optimization process.

$$
F n e t(t) \geq F 1(t) ; F 2(t) ; F 3(t) ; F 4(t)
$$

From (5) and (6), can get:

$$
\begin{aligned}
& M_{n e t}=F_{1} \cdot x_{1} \cdot \cos \theta_{1}+F_{2} \cdot x_{2} \cdot \cos \theta_{2}+F_{3} \cdot x_{3} \cdot \cos \theta_{3}+ \\
& F_{4} \cdot x_{4} \cdot \cos \theta_{4}
\end{aligned}
$$

At the balance point condition where $\left(x_{c}=x_{o}=0\right)$, (9) will be $\left(M_{n e t}=0\right)$. The mutual force between each plane disc and coil is the vertical displacement and magnetic suspension force. The working parameters are $\mathrm{F}$ which is weight force of the body, $\mathrm{x}$ is the gap distance between the body surface, and the Maglev coil, $\mathrm{v}$ is the body velocity, $\mathrm{t}$ is the time representation and $\mathrm{M}$ is the body mass.

\section{B. Magnetic Sub-model}

Maglev trains are powered by magnetic levitation technology. The magnets have two opposite-pole parts able to attract, in the other effect there are two like-pole magnets will push apart. The capacity of the magnet based on force attract came from ferromagnetic and responses by the air gap position. Strength of magnetic force is change based on the different shape design of the magnet. The optimal force can be presented by [11]:

$$
F=\frac{B^{2} A}{2 \mu_{0}}
$$

Where $\mathrm{F}$ is the electromagnetic attractive force in Newton; $\mathrm{B}$ is the magnetic flux in Tesla; $\mathrm{A}$ is the cross section area of the pole face in square meters; $\mu_{0}$ is the free space permeability $\mu_{0}=4 \pi \times 10^{-7} H M^{-1}$.

Based on the magnitude of flux density in the core, the core area, the magnetizing field $(\mathrm{H})$ in the air-gap, the electromagnet current must be calculated by [12]:

$$
i=\frac{m m f}{N}
$$

It is necessary to use the current (i) (or voltage) capability which reflects the magnet power amplifier magnitude. 


\section{Electrical Sub-model}

Transport systems can be represented by a set of dynamical equations obtained from the Euler-Lagrange energy method. The electrical circuit of the system presents the relation between current and voltage of the circuit based on Kirchhoff Voltage Law. Voltage drop on the resistor is R.i(t) and inductor is $\mathrm{L}((\mathrm{di}(\mathrm{t})) / \mathrm{dt})$. Applied voltage in each coil of the system is equal to the summation of the voltage of the resistor and the inductor.

$$
V(t)=R \cdot i(t)+L\left(\frac{d i(t)}{d t}\right)
$$

From the present equations, the coil inductance value is computed by using the effective gap area instead of the actual center-pole area to avoid the errorse [13]. The inductance of the coil will be independent of I. Thus, $L(x)$ will depend only on the geometry. The electromagnetic inductance of the coil is assumed to have the form of Fig. 2.

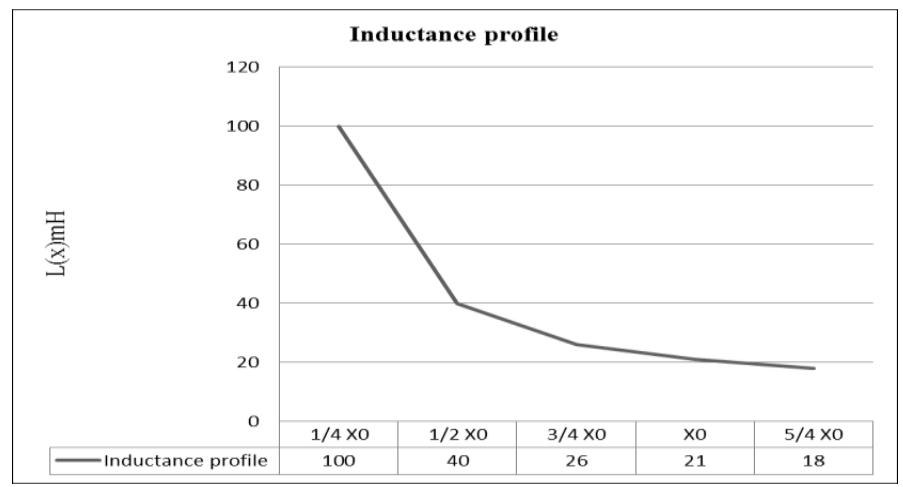

Fig. 2. Variation of the inductance of the coil with position.

From the above figure, the conclusion is:

$$
L(x)=L_{s}+L_{0}\left(\frac{x_{0}}{x}\right)^{2}
$$

The present result quid to find out the force constant which represent the key factor in Maglev system calculations. The force constant in the present methodology was used for a plan Maglev which considered one value work with four coils in parallel with the same time consumption. The methodology to gain this value used the experimental tests correlated with the derivative of theoretical equations to determine the inductance response. This method represents a novel trail for finding the force constant value for this case.

\section{PHASE LEAD COMPENSATION CONTROL}

Phase Lead Compensation Control can be expressed as a classical control category. It is commonly used feedback controller, for that it is widely applied in industrial control systems [14]. This control system is working based on the calculations of the error value, trying to reduce the error percentage by adjusting the controller parameters. The general form of this controller formulated is as follows:

$$
G_{c}(S)=K_{c} \cdot \alpha \frac{T s+1}{\alpha T s+1}=K_{c} \frac{s+\frac{1}{T}}{s+\frac{1}{\alpha T}}
$$

$$
G_{c}(s)=K_{c} \cdot \frac{s+Z}{s+P}
$$

Fig. 3 observe the three close loop poles gained from the root locus. It is clear that the poles position are in the left half side which represent the complex plane, that's indicate to the stability of the system based on the Lead controller as shown in Fig. 4.

As shown in Fig. 5 and 6 observe the selected point which gives a stable close loop poles. This represent step system parameters with less rise time of (0.297) sec. and settling time of (0.566) sec. which represent a satisfactory for the system without overshoot. The plot of step response observes the controller succeed to suppress the disturbances and there is a need to scale down the reference point in order to catch up with step response. From the presented result, the researcher concluded that the phase lead controller gain a good response due to the trajectory tracking with no overshoot.

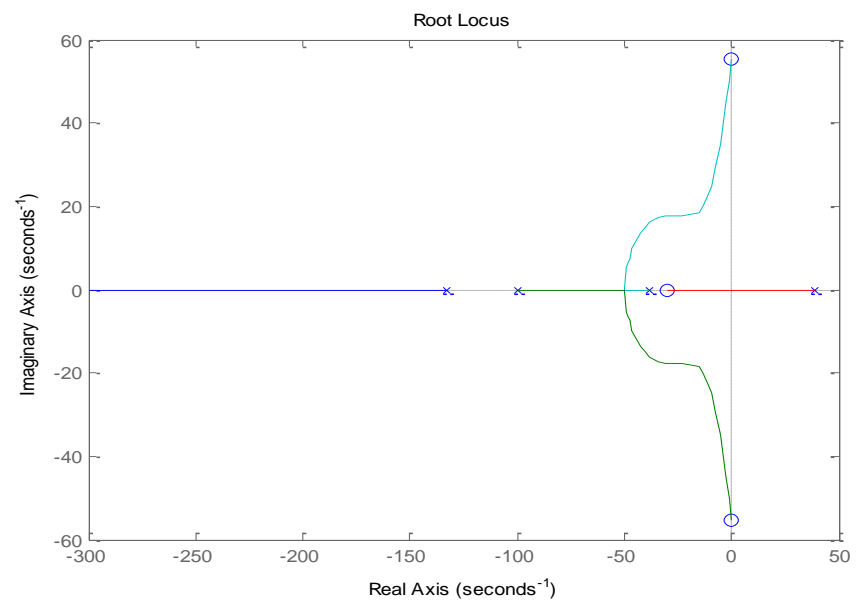

Fig. 3. Three close loop poles.

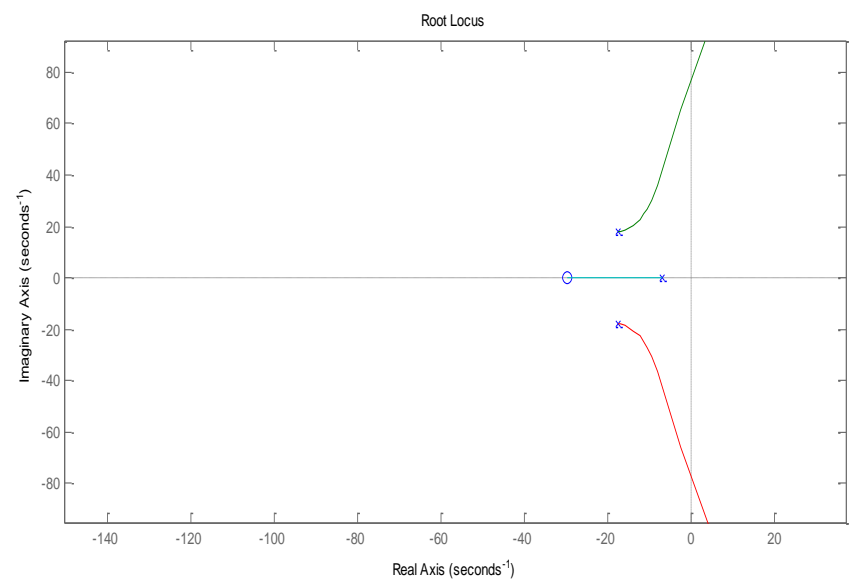

Fig. 4. Root locus phase lead controller. 


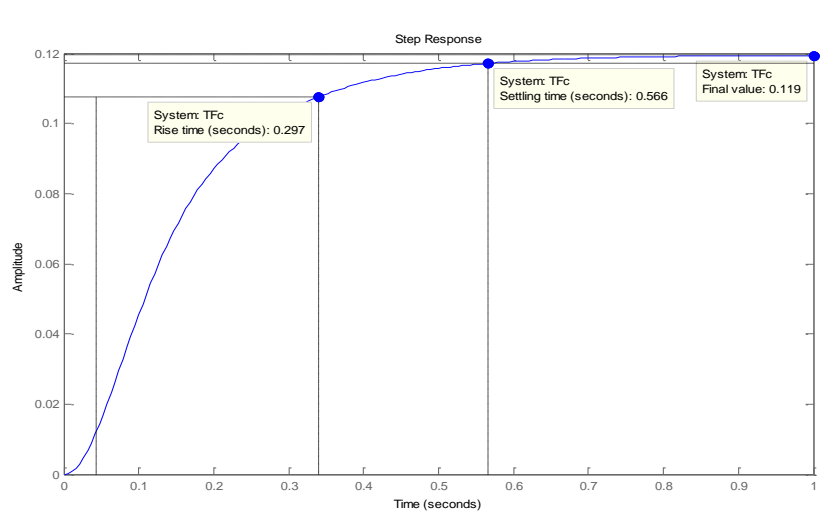

Fig. 5. System step response.

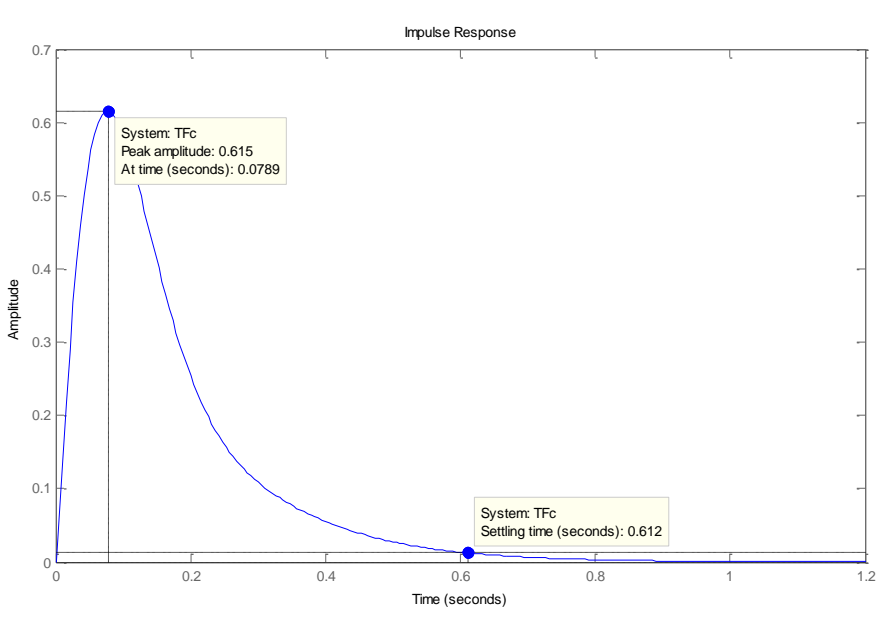

Fig. 6. System impulse response.

In order to measure the speed of the system, the settling time and the delay tine represent the main needed parameters. The increasing in undamped natural frequency causes an increase in in the system speed. Also, when the damping ratio increases cause a reduction in the maximum overshoot, while the delay time also increases. This situation causes a sluggish in system response [15].

\section{HARDWARE CONTROL UNIT}

The hardware control unit is presented in details in previous sections. In this section, the researcher will describe the method to implement this essential part. The SIMLAB contains set of input representations and output representations, the SIMLAB hardware will be connected with both of the Maglev prototype and the MATLAB Simulink which enable the system to control and operation. Proximity sensors are specific devices that enable to measure the air gap distance. There are many types of these sensors such as laser, inductive, resistive, hall-effect and IR sensors. In the present system, the hall-effect sensor has been used to detect the distance air gap measurement. The sensor position in the present Maglev system is in the bottom of the coil. The unique feature of this type of sensors encourages the researchers and producers to use it in many fields such as the aircrafts, automobile and medical machines. The operation concept of the sensor is to detect the magnetic field of the coils. The output voltage is induced on the output terminals of the sensor. This mechanism depends on the intensity of the magnetic field.

\section{RESULTS AND DISCUSSION}

The position of the suspended object considered as a functional parameter in the force levitation action. The point of equilibrium is considered based on the gravitation, electromagnetic field which always must be zero net force. If the object position represents an input representation to the Maglev controls system. If the right position detected and feed into the control formula, the system can deal the variations of the electromagnets strength and the object can levitated easily. Based on this concept, this section presents the experimental tests that done by the system prototype. The tests signal input representation involves three different standard signals. The first one is the sine wave testing signal. The results are shown in Fig. 7. It represents a fundamental test signal which is used in electronic test procedures. It indicates a smooth and stable sequence input representation to the Maglev system. The second signal is the square wave testing signal. The square wave represents a signal with rich in harmonics. All the harmonics is odd based on the symmetrical shape of the signal. The third signal is the sawtooth wave signal. The sawtooth wave is one of the most important signals in electronic tests. All the tests results are described in the next section. The Lead controller observes the results of the applied signals.

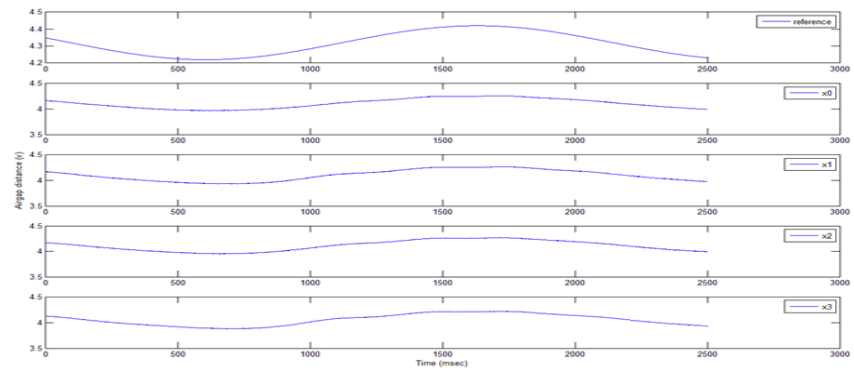

Fig. 7. Sine wave signal applied on plane based on lead control system.

The results indicate that the system is stable and responds homogeneously. It is clear from the signal response that all points respond based on the same input wave. This result indicates that the system able to deal with the force reaction and the dynamic moment of the plane. This result mimics the real Maglev cart movement under crispy land when the load changes dynamically based on the train cart movement up and down.

The next signal test is the square wave. The testes done based on the same three types of control system as mentioned before. The results are present in Fig. 8. 


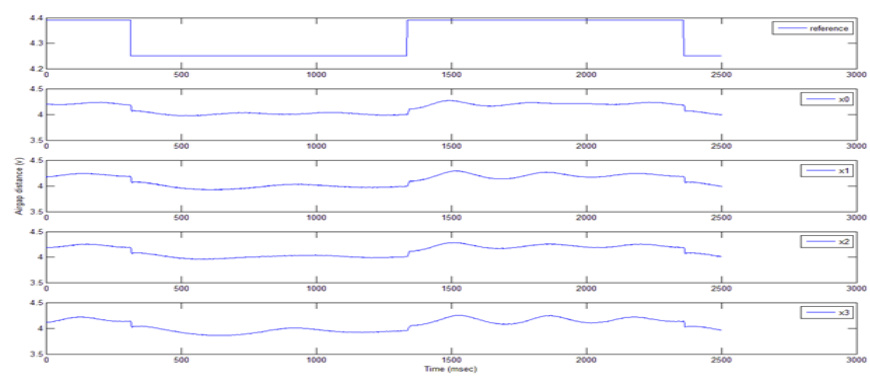

Fig. 8. Square wave signal applied on plane based on lead control system.

The results of the square wave tests indicate that the system respond is also stable. The system also able to absorb the force reaction and the dynamic moment in the load points to work similar to the absorb damper. This test presents the ability of the system to respond like the spring and damper in mechanical systems. This result represents one of the important contribution results of the present work.

The final test in the point effect on plane Maglev system is the sawtooth wave signal. The results are shown in Fig. 9.

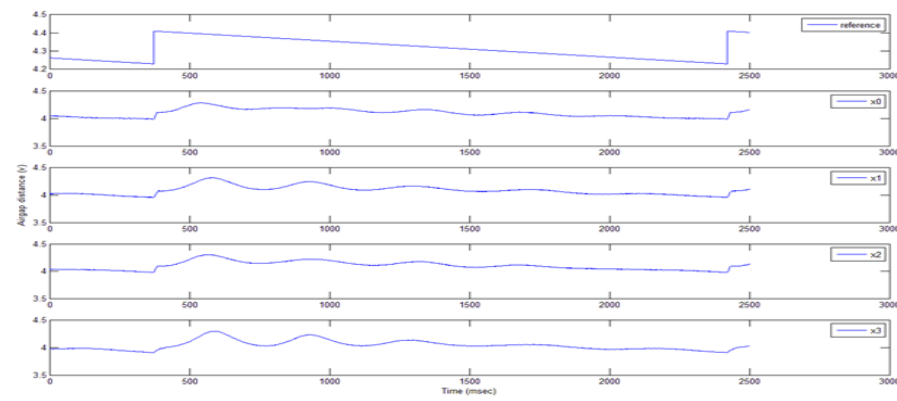

Fig. 9. Sawtooth wave signal applied on plane based on Lead control system

The results show that the Maglev system prototype which are designed and produced on this research presents significant results.

\section{CONCLUSION}

The concept of Maglev system is to suspend the object with no support other than magnetic fields. The effects of the gravitational acceleration and the other effects will be handled by magnetic forces. Therefore, the main direction in this paper is to investigate the magnetic levitation properties which involve sufficient upward force to counteract gravity. The system respond was significant to the three types of applied waves. The results of applying a wave signal effect on the full plane in the present prototype observed the system is stable and responds homogeneously. It is clear from the signal response that all points respond based on the same input wave. This result indicates that the system is able to deal with the force reaction and the dynamic moment of the plane. In terms of directions for future research, further work could be used as other control method such as PID or LQR control.

\section{REFERENCES}

[1] S. A. Shaheen and R. Finson, "Intelligent Transportation Systems," 2004.

[2] N. D. Pandey, M. Kumar, and P. Tiwari, "Analysis of Magnetic Levitation and Maglev Trains," vol. 3, no. 12, pp. 108-112, 2016.

[3] N. F. Al-Muthairi and M. Zribi, "Sliding Mode Control of a Magnetic Levitation System," Math. Probl. Eng., vol. 2, no. October 2003, pp. $93-$ 107, 2004.

[4] S. Banerjee, D. Prasad, and J. Pal, "Design, implementation, and testing of a single axis levitation system for the suspension of a platform," ISA Trans., vol. 46, no. 2, pp. 239-246, 2007.

[5] Y. Lee, J. Yang, and S. Shim, "A new model of magnetic force in magnetic levitation systems," J. Electr. Eng. ..., vol. 3, no. 4, pp. 584592, 2008.

[6] Y. Khemissi, "Control Using Sliding Mode Of the Magnetic Suspension System," no. 3, pp. 1-5, 2010.

[7] Liu and J. Zhang, "Design of second-order sliding mode controller for electromagnetic levitation grip used in CNC," Proc. 2012 24th Chinese Control Decis. Conf. CCDC 2012, vol. 2, no. 1, pp. 3282-3285, 2012.

[8] Xing, B. Kou, C. Zhang, Y. Zhou, and L. Zhang, "Levitation force control of maglev permanent synchronous planar motor based on multivariable feedback linearization method," in Electrical Machines and Systems (ICEMS), 2014 17th International Conference on, 2014, pp. 1318-1321.

[9] Unni, A. S. Junghare, V. Mohan, W. Ongsakul, and E. Fos, "PID , Fuzzy and LQR Controllers for Magnetic Levitation System," vol. 0, no. September, pp. 14-16, 2016.

[10] H. Zhu, T. J. Teo, and C. K. Pang, "Design and Modeling of a SixDegree-of-Freedom Magnetically Levitated Positioner Using Square Coils and 1-D Halbach Arrays," IEEE Trans. Ind. Electron., vol. 64, no. 1, pp. 440-450, 2017.

[11] P. K. Biswas and S. Bannerjee, "Analysis of U-I and U-U Type Rail and Actuator Used in Electromagnetic Levitation System Using FEM Software," vol. 2, no. 5, 2012.

[12] P. K. Biswas and S. Banerjee, "ANSYS simulation based comparative study between different actuators and guide-ways used in DC electromagnetic suspension systems," Int. J. Electr. Eng. Informatics, vol. 4, no. 2, pp. 217-230, 2012.

[13] P. Education, "Section 5," pp. 109-135.

[14] K. Sailan and K. Kuhnert, "DC motor angular position control using PID controller for the purpose of controlling the hydraulic pump," Proc. Int. Conf. Control. Eng. ..., vol. 1, pp. 22-26, 2013.

[15] Sintayehu, "MAGNETIC LEVITATION ON School of Graduate Studies MAGNETIC LEVITATION ON," no. April, p. 112, 2007. 